\title{
Application of posture recognition in intelligent riding auxiliary equipment
}

\author{
Tian Jiaxin ${ }^{1}$ \\ Information Engineering Institute \\ Beijing Institute of Fashion Technology \\ Beijing, China \\ 617724382@qq.com \\ Du Yang ${ }^{2}$ \\ Information Engineering Institute \\ Beijing Institute of Fashion Technology \\ Beijing, China \\ 18310236998@163.com
}

\author{
Guo $\mathrm{Fei}^{3^{*}}$ \\ Digital media and interactive Key Laboratory \\ Beijing Institute of Fashion Technology \\ Beijing, China \\ lunwen95@163.com \\ Peng Shengqiong ${ }^{3}$ \\ Digital media and interactive Key Laboratory \\ Beijing Institute of Fashion Technology \\ Beijing, China \\ ysylab@bift.edu.cn
}

\begin{abstract}
For cyclists, whether the purpose of riding is health or stimulation, safety always ranks first. In order to enable users to pay more attention to the road condition and not be distracted during the riding process, this topic pursues conciseness and clarity in the functional design. By using the posture recognition function, users can only move their shoulders to the left or right and lean forward slightly during the riding process, indicator lights fixed to the corresponding parts of the body can be lit to inform other drivers and pedestrians of driving intentions, functional and intelligent enough. In software simulation and field testing, good recognition results have been achieved.
\end{abstract}

Keywords-posture recognition; riding safety; neuron; MATLAB simulation

\section{INTRODUCTION}

With the growing maturity of mobile interconnection technology and the perfect combination of technology and clothing, the field of intelligent wearable is shining brilliantly. Intelligent riding apparel has made great progress in fabric, function and modeling, [1]. However, in the design of smart clothing with technology as the key element, technology will affect the function and overall performance of smart clothing, as well as the interaction and fashion of smart clothing. Therefore, we should make good use of technology as a double-edged sword, while ensuring the wearability of clothing, hide as much as possible the electronic components added to smart clothing [2].

The safety of urban traffic is more important, especially when riding at night. In recreational cycling equipment, cycling clothing, helmet, gloves, glasses and other equipment can bring good protection for users. The intelligent riding auxiliary device based on posture recognition is also a safety device. On top of comfortable and breathable bicycle clothing fabrics, LED indicator lights are added. By means of a six-axis attitude sensor and gyroscope mounted on the rider's spine, the turning light on the sleeve automatically lights up when turning left or right, just like the turning light on the car. When encountering deceleration, the brake lights behind the clothes are on. The drivers and passers-by can see the cyclists at any time and take corresponding evasive measures to improve the safety factor of riding at night.

\section{OVERALL FUNCTIONAL DESIGN}

The aim of this project is to improve the safety factor of night cycling, ensure the personal safety of cyclists as far as possible, and bring better sports experience for cyclists. The left-right steering and brake light of the device can light the reminder light according to the specific body posture of the rider (shoulder movement and body forward), and inform the driver of the rider's intention in advance, so as to ensure the safety of night riding.

After investigation and measurement, the female arm circumference was $20 \sim 27 \mathrm{~cm}$ and the male arm circumference was $30 \sim 40 \mathrm{~cm}$. In this project, the length of intelligent steering lamp belt is designed to be $20 \mathrm{~cm}$, and the length of decelerating lamp belt is designed to be 15 $\mathrm{cm}$. The width of both is $6 \mathrm{~cm}$. The LED lamp belt is fixed by velcro which is easy to wear and tear.

\section{LEARNING AND TRAINING OF MOTION DATA}

\section{A. Curie Neuron Kit Suite}

The general steps of using Curie Neuron Kit to do learning and training of action data are as follows: 
- The Bluetooth 4.0 module on board is used to communicate with the Bluetooth device on the computer, and the data between Arduino 101 [3] and Bluetooth adapter is sent and received through the serial port monitor, so as to monitor the data of 6-axis accelerometer and gyroscope [4].

- reads and writes motion data and burns corresponding programs. Each action is repeated 20 times. After power failure, the SD card is connected to the computer. The data of the acceleration sensor can be seen in the. CSV file.

- training and recognition of movement data. The corresponding neuron learning program is written on the development board. When Bluetooth matches automatically, Curie learns the processed data automatically.

- Through the serial port monitor to mark the corresponding position of the program, the experimenter can judge the accuracy of action recognition.

\section{B. Data processing}

The data processing steps are as follows:

- The preset action data are collected one by one with the six-axis acceleration sensor of Inter Curie, and stored on the SD card in CSV format.

- Copy the data stored in SD card to the computer, open the. CSV file, then select the experimental data, insert the line chart, and filter out the irregular waveform.

- Sampling and de-sampling of data;

- Copy the processed data back to SD card to let Inter Curie learn these actions.

- Use the same steps to train, identify and learn different behavior actions.

1) read and write motion data

a) materials required

Inter Curie development board *1

USB data line $* 1$

Micro SD card *1

Micro SD card reader *1

SD card read and write storage module *1

When collecting data, we should keep the consistency of action amplitude and speed as far as possible, so as to facilitate subsequent data processing.

\section{b) read and write MicroSD card data}

SD card development library provides a sample of SD card file reading and writing, which can be found in "example-SD_ReadWrite".

Connect 5V power supply to Inter Curie, start shoulder left movement, power off after about 20 repetitions, read the data collected from SD card to the computer, then double-click open.csv file:

\begin{tabular}{|r|r|r|r|r|}
\hline & A & B & C & D \\
\hline 1 & 0.25 & 0 & 1.01 \\
\hline 2 & 0.25 & 0 & 1 \\
\hline 3 & 0.25 & 0 & 1 \\
\hline 4 & 0.25 & 0 & 1 \\
\hline 5 & 0.25 & 0 & 1.01 \\
\hline 6 & 0.25 & -0.01 & 1.01 \\
\hline 7 & 0.24 & -0.01 & 1 \\
\hline 8 & 0.24 & 0 & 1.01 \\
\hline 9 & 0.24 & 0 & 1.01 \\
\hline 10 & 0.24 & 0 & 1.01 \\
\hline 11 & 0.24 & 0.01 & 1.01 \\
\hline 12 & 0.24 & 0 & 1.01 \\
\hline 13 & 0.24 & 0 & 1.01 \\
\hline 14 & 0.24 & 0 & 1.02 \\
\hline 15 & 0.24 & 0 & 1.02 \\
\hline 16 & 0.24 & 0 & 1.02 \\
\hline
\end{tabular}

Fig. 1. Value of the six axis sensor in the.Csv file

The $\mathrm{A}, \mathrm{B}$ and $\mathrm{C}$ columns in the above figure are the data collected from $\mathrm{X}, \mathrm{Y}$ and $\mathrm{Z}$ axis respectively.

Select all the collected action data in the table and insert a broken line diagram as shown below:

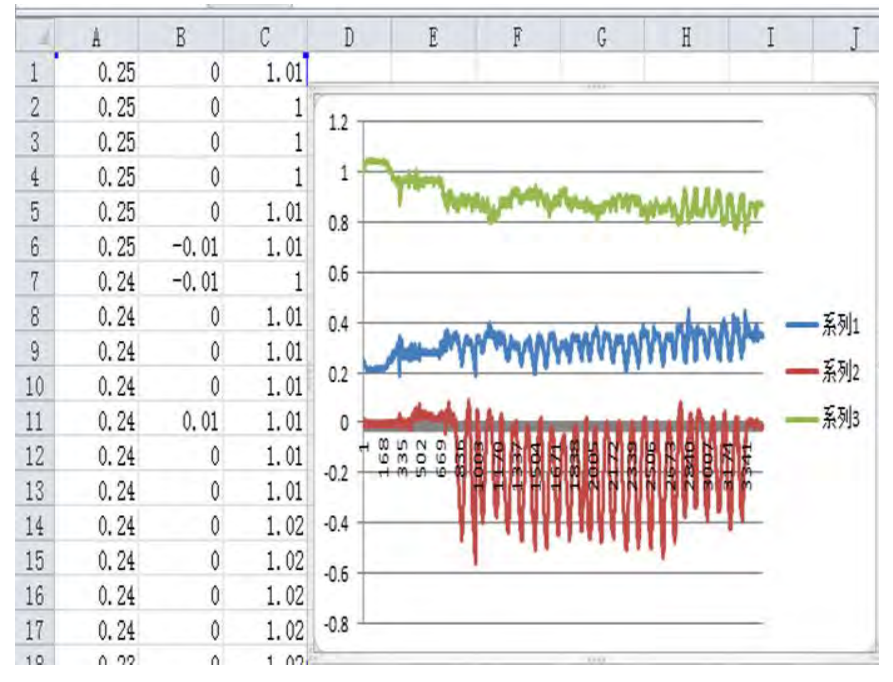

Fig. 2. Line chart for generating raw data of $\mathrm{X}, \mathrm{Y}$ and $\mathrm{Z}$ axes.

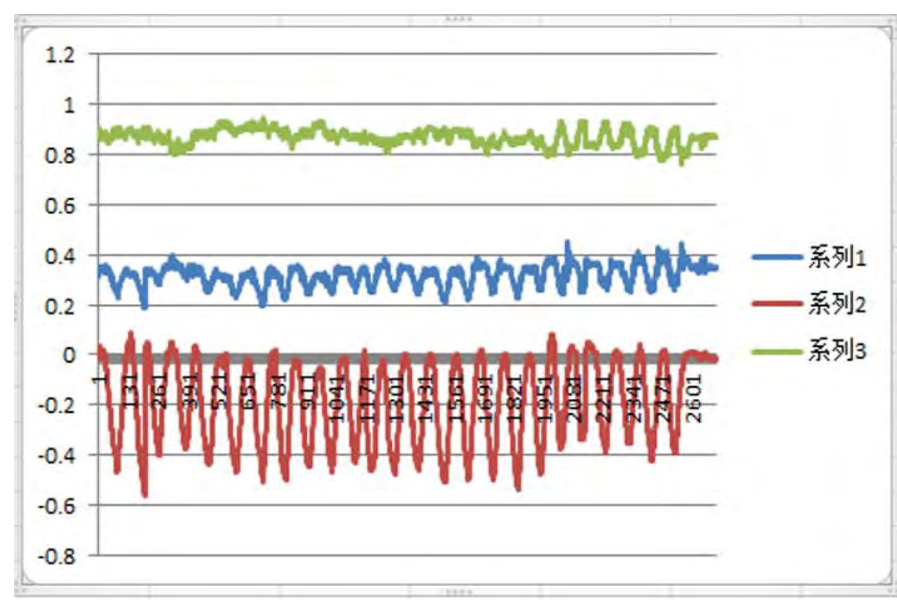

Fig. 3. Filtered line chart 


\section{2) data processing}

Each waveform cycle indicates that a preset action is completed.

Cut out the irregular clutter, just delete the data in the corresponding table, and the processed waveform is as Fig.3.

Processing other action data in the same way and running QriNeuronPipeline.exe after modifying the configuration in the config.csv file for data processing, the results are as follows:

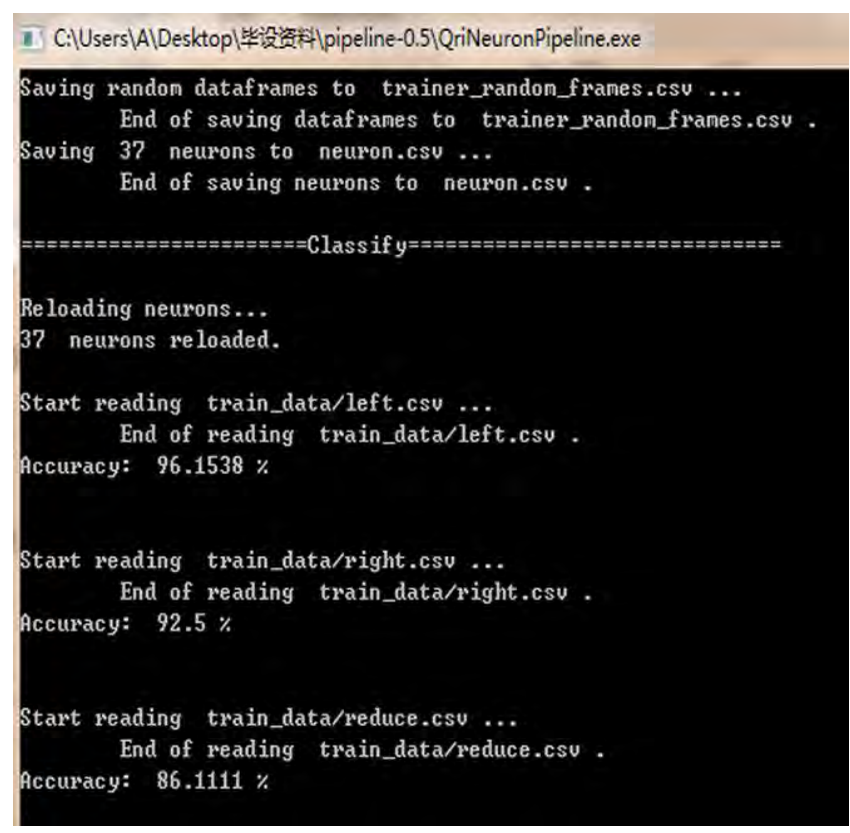

Fig. 4. Data processing results

The results show that the recognition rates of the three actions are $96.1538 \%, 92.5 \%$ and $86.1111 \%$ respectively, which represents the accuracy of Curie recognition.

The processed data will generate the "neurons.csv" file and then save the file to SD card. Finally, "curie-classfiybl" is burned into Inter Curie and inserted into SD card. After 30 seconds, Curie can learn and recognize automatically.

\section{SOFTWARE SIMULATION AND OPTIMIZATION}

\section{A. MATLAB simulation}

The collected three-axis data are sorted into the "dataread.txt" text file, which contains four columns of data, namely, x-axis, y-axis, Z-axis and category. The amount of input in the network is the acceleration value in different directions, and the output quantity is the result of classification. The collected data are simulated by Matlab [6], and the process is as follows:

- Read training data: read the required data;

- Normalization of eigenvalues: using functions to process data between 0 and 1 ;
- Construct output matrix: determine the size of the output matrix by category information in the data.

- Create a neural network: create the neural network to be used in this project.

- Set up training parameters: set some parameters of the network, so that it can better train and learn.

- Start training: after completing the whole training process, feedback the corresponding information.

In the above process, the most important process are data preprocessing and network parameter setting. In the setting of network parameters, the selection of parameters has a great impact on the overall performance of the network [4]. The number of hidden layer nodes will not have a significant impact on the recognition rate, but when the number of nodes is too large, the amount of network operation will increase dramatically, thus reducing the efficiency of the overall network learning. The activation function has a significant effect on the recognition rate and convergence speed of action data. In infinite approximation of high-order curves, the accuracy of Stype function is much higher than that of linear function, but the computational complexity is also much larger than that of the latter. The choice of learning rate in parameter setting is also critical. The choice of learning rate will affect the convergence and convergence effect of the network [5]. When the learning rate is set on a small scale, it can ensure that the network is convergent, but the convergence speed is relatively slow. If the learning rate is set on a large scale, the network is likely not convergent, which will affect the recognition effect. Only by setting these parameters reasonably can the training and learning of the data of the neural network get better results.

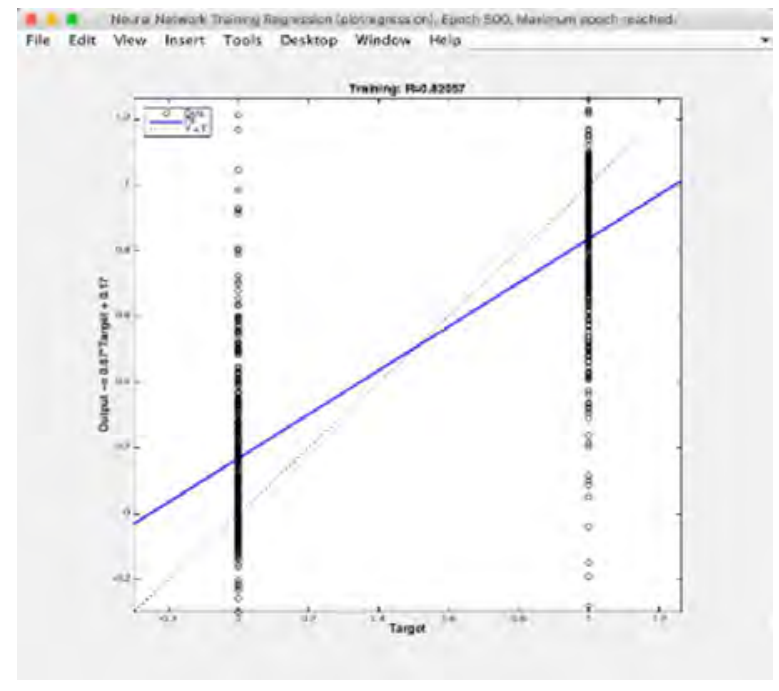

Fig. 5. Fitting rate

As shown in the figure above, the data fitting rate is $82.057 \%$. In this project, the acceleration value changes within a small range. After data normalization, most of the fitted data are distributed between 0 and 1 , and the vertical lines with abscissa 0 and 1 are the most dense at the intersection of the fitted oblique lines, that is, here is the better part of data fitting. 
According to the analysis, although the data obtained from the acceleration sensor is disorderly and real-time, it is also convergent. So the BP neural network algorithm burned in the main control board is effective for the data of acceleration sensor, so this kind of action data can be trained and learned by using the Inter Curie main control board.

\section{B. interrupt procedure}

Only when the prompt lamp flickers, the stop time of the system will not affect the recognition of the next action, that is, it will not cause confusion in the program, so the interrupt program is added in the program segment of the light. This topic designs a software switch to simulate external interruption.

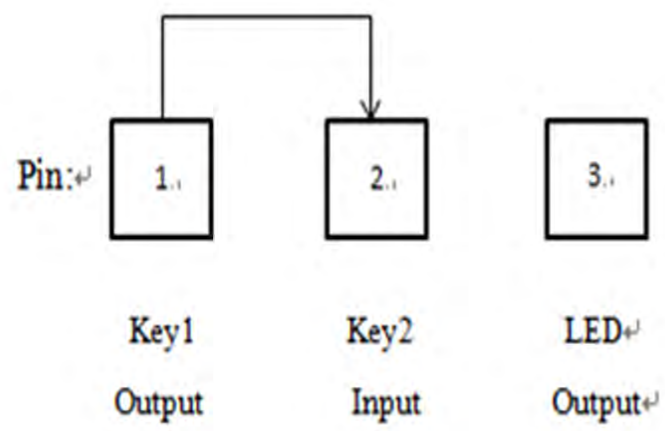

Fig. 6. Software switch

The initial values of pin1, Pin2 and PIN3 are all set to low level. When the action type is judged, the value of Pin1 is set to high level. At this time, the value of Pin2 read to Pin1 becomes high level. When the value of Pin2 is high level, it is interrupted and the corresponding Led lamp is lit.

\section{IMPLEMENTATION OF HARDWARE SYSTEM}

\section{A. Design and implementation of switch circuit}

This project has designed a switch circuit, which can control the lighting of the lamp belt through the main control panel. The transistor used is type NPN C9031.

If only transistor is added to the circuit, the lamp belt can be lit up normally, but the triode is very easy to generate heat. Therefore, it is necessary to add resistors to solve the problem of triode heating[7]. The testing process is as follows:

- If resistance is added to both the base and collector of the transistor, the voltage added to the lamp band is insufficient, resulting in insufficient brightness of the lamp band.

- If only the resistance is added to the basic stage of the transistor, the brightness of the lamp band can meet the expected requirements, but the phenomenon of transistor heating still exists.
- If only a resistor is added to the collector of the transistor, the lamp band will be bright enough, and the transistor will not have obvious heating phenomenon after a long period of electrification.

Finally, a resistor of $4.7 \mathrm{~K}$ is added to the collector of the transistor, and a simple switch circuit is realized. The circuit diagram is as follows:

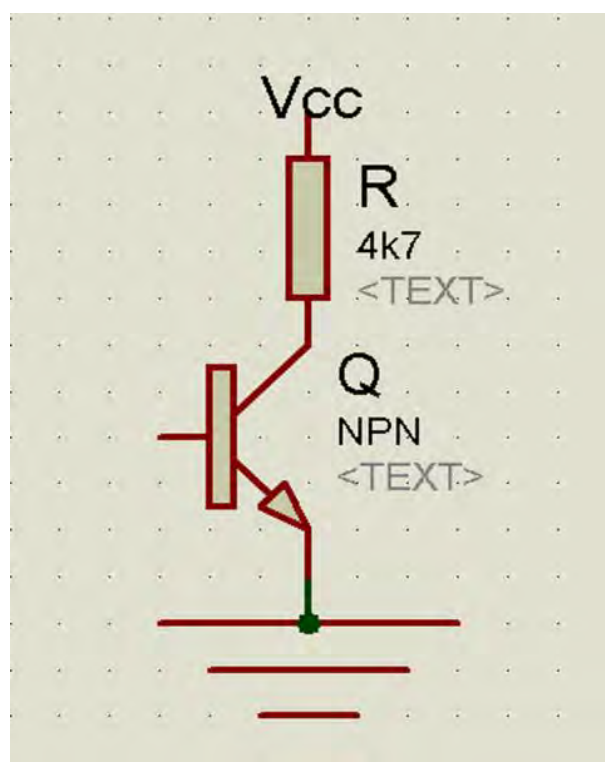

Fig. 7. Triode switch circuit

\section{B. Integrated hardware system}

The schematic diagram of the circuit is as follows:

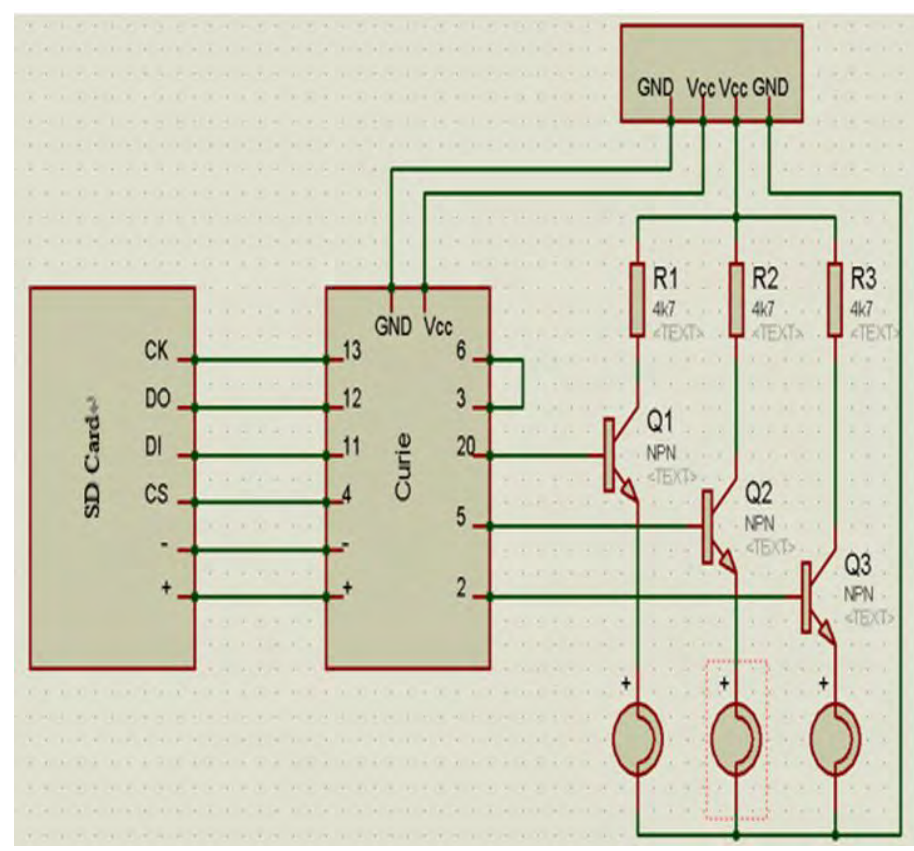

Fig. 8. Schematic diagram of the whole hardware circuit

The distribution of pins in Curie Nano is as follows:

SD card module: pin13, pin12, pin11, pin4;

Software keypad: pin3+pin6; 
Right turn indicator light: pin5;

Left turn indicator: pin20;

Deceleration indicating lamp: Pin2;

Power output: 5V+GND

\section{CONCLUSION}

Combining six-axis accelerometer, gyroscope sensor and 128 neurons in Inter Curie Nano main control board, the design and development process of riding aids based on attitude recognition is realized. It can basically realize the preset functions of turning and decelerating hints, but the recognition rate is not good enough to be applied to actual cycling. The actual test shows that the left and right steering can be accurately identified, and the recognition rate is higher than $90 \%$. But recognizable movements are larger than the preset ones, because the shoulder movement is centered around the spine, and the spine movement is smaller than the movement during the test. The recognition rate of the deceleration indicator corresponds to the forward movement of the body is low. And sometimes it can be mistakenly identified as turning left or right. It should be that in the process of data processing, the extraction of eigenvalues is not good enough, and there is a cross between the eigenvalues, which leads to the identification is not high enough.

\section{ACKNOWLEDGMENT}

This article is one of the research achievements of the 2017 Beijing Municipal Education Commission Science and Technology Program on the surface of the project (SQKM201710012006), Innovative design of garment ergonomics and apparel functions Beijing key Laboratory (KYTG02170202), and Digital media and interactive Key Laboratory of Beijing.

\section{REFERENCES}

[1] Xie Junxiang, Zhang Lin. Intelligent wearable device and its application [J]. China medical device information, 2015, (03): 1823.

[2] Li Jing. Bicycles: intelligent cycling has been on the way to [N]. consumer daily.2014-11-25 (A02).

[3] Zhao Yingjie. Perfect illustration. Introduction to Arduino interactive design. Beijing: Science Press, 2014.7.

[4] Edited by Chen Wenbai. Principles and Practice of Artificial Neural Networks. Xi'an: Xi'an University of Electronic Science and Technology Press, 2016.01.

[5] Fei Si technology research and development center, MATLAB6.5 aided image processing, electronic industry press, 2003.1

[6] Chen Ming, principles and examples of neural network, Tsinghua University press, 2013.3.

[7] Sun Dejun editor in chief. Easy to learn and play Arduino. Arduino.: Chemical Industry Press, 2016.10 